RESEARCH ARTICLE

\title{
Clinical Risk Factors and Multi-Antimicrobial Resistance Pattern in Community-Acquired Outpatient Urinary Tract Isolates of Escherichia Coli
}

\author{
M Nadeem Ahmed ${ }^{1,2^{*}}$, Debby Vannoy ${ }^{1}$, Ann Frederick ${ }^{1}$ and Xuan $\mathrm{Bi}^{2}$ \\ ${ }^{1}$ Carle Foundation Hospital, Urbana, USA \\ ${ }^{2}$ University of Illinois, Urbana, USA
}

*Corresponding author: M Nadeem Ahmed, MD, PhD, Pediatric Hospital Medicine, Carle Foundation Hospital, 611 West Park Street, Urbana, II 61801, USA, E-mail: nadeem.ahmed@carle.com

\begin{abstract}
Introduction: Urinary tract infections (UTIs) are one of the most commonly treated bacterial infections in ambulatory care clinics and emergency departments (ED). The management of UTIs has been complicated by the emergent resistance to most commonly prescribed antibiotics causing increased patient morbidity, cost of reassessment and retreatment, and rates of hospitalization.
\end{abstract}

Objective: To evaluate multi-antimicrobial resistance pattern of Escherichia coli (E. coli) urinary isolates and the risk factors associated with commonly prescribed antibiotics in emergency department and primary care clinics.

Method: This is a cross-sectional study of patients 18 to 65 years of age reported to have $E$. coli positive UTIs whose medical and laboratory records were systematically reviewed.

Results: Overall, $37.7 \%$ E. coli urinary isolates were resistant to ampicillin, $18.3 \%$ to trimethoprim/sulfamethoxazole (TMP/SMX), and $7.8 \%$ to ciprofloxacin. About $21 \%$ isolates were resistant to 2 or more antibiotics. Ciprofloxacin-resistant $E$. coli isolates from outpatient urine sample were frequently resistant to ampicillin (81.5\%), and TMP/SMX $(58.2 \%)$. The concurrent resistance rate of ciprofloxacin was about 8 times more frequent $(24.8 \%$ vs. $3.1 \%)$ than nitrofurantoin among TMP/SMX-resistant E. coli urinary isolates. Patients with histories of genitourinary abnormalities were 1.59 times $(C l 1.27-1.98)$ more likely have $E$. coli isolates resistant to TMP/SMX, and 2.35 times more likely (Cl 1.79-3.09) to ciprofloxacin. Diabetic patients were at increased risk for resistance to TMP/SMX (OR 1.37, Cl 1.141.65) and ciprofloxacin (OR 2.51, Cl 2.00-3.16). Obesity is significantly associated with ciprofloxacin resistance (OR 1.68, Cl 1.34-2.09). TMP/SMX and ciprofloxacin resistance rate increased gradually with the number of previous UTIs, hospitalizations, and antibiotic prescriptions.
Conclusions: Ciprofloxacin resistant isolates of $E$. coli from urine were frequently multi-drug resistant and TMP/SMX can induce ciprofloxacin resistances. In addition to demographic factors, history of genitourinary abnormalities, diabetes, obesity, number of hospitalizations, previous diagnosis of UTIs, antibiotic prescriptions in previous 6 months are risk factors for antimicrobial resistance.

\section{Keywords}

Urinary tract infection, Antibiotic resistance, Antibiotics, Risk factors, Ciprofloxacin, Fluoroquinolones, Ampicillin, Trimethoprim-sulfamethoxazole, Cefazolin, Obesity, Diabetes, Escherichia coli

\section{Introduction}

The management of Escherichia coli (E. coli) driven urinary tract infections (UTIs) has been complicated by the emergence of resistance to commonly prescribed antibiotics causing increases in patient morbidity, cost of reassessment and retreatment, and rates of hospitalization $[1,2]$. While resistance to amoxicillin has been established for years, trimethoprim/sulfamethoxazole (TMP/SMX) resistance became more prevalent in recent years. Studies have shown that TMP/SMX resistance rate varies by region ranging from $18 \%$ to $50 \%$ worldwide and from $18 \%$ to $25 \%$ in North America [36]. Recently revised published guidelines by the Infectious Disease Society of America (IDSA) recommended $\mathrm{TMP} / \mathrm{SMX}$ therapy should not be used as empiric therapy in regions where the prevalence of TMP/SMX resistance rates exceed 20\% [7]. Although emphasis has 
been given to prescribe more narrow-spectrum antibiotics for treating simple uncomplicated UTIs whenever possible, concerns about resistance have resulted in escalating use of broad-spectrum antibiotics, particularly cephalosporins, and fluoroquinolones. In recent years overall use of fluoroquinolones, particularly ciprofloxacin in ambulatory care, has dramatically increased [89]. Clinicians and researchers already began to observe an increasing trend in $E$. coli resistance urinary isolates for quinolones in addition to ampicillin and TMP/SMX $[5,6,10]$. The focus of this study was to identify common risk factors as well as the distribution and characteristics of multi-antimicrobial resistance patterns of commonly prescribed antibiotics among $E$. coli infected UTI patients in community ED and outpatient clinics.

\section{Methods}

\section{Study design}

We performed a retrospective cross-sectional study examining urinary $E$. coli isolates of patients aged 18 to 65-years-old collected in ED and primary care clinics. Laboratory data were matched with the corresponding individual's demographic information and medical record. The study was conducted at a university affiliated community hospital. Primary care clinics were defined as non-specialty care clinics within the Internal Medicine and Family Medicine departments. The medical records of these identified patients being evaluated were then reviewed to obtain demographics and clinical data. All medical information was in electronic format. Patients were excluded if their records were not available, if urine culture grew more than one organism, or if a urine contamination was suspected. Voided cultures with greater than $100,000 \mathrm{CFU} / \mathrm{mL}$ and cultures collected via catheterized specimen with greater than $10,000 \mathrm{CFU} / \mathrm{mL}$ were not considered contaminants [7]. Urine cultures positive for $E$. coli that were drawn from patients visiting in the primary care clinics and the ED from January 1, 2011 to December 31, 2012 were eligible for inclusion in the analysis. $E$. coli isolates with intermediate susceptibility were not classified as being resistant. The study was approved by the Carle Institutional Review Board (IRB).

\section{Measurements variables}

Demographic data included patient age, date of birth, gender, race, and insurance status. Clinical data consisted of date of encounters, height, weight, methods of specimen collection, urinalysis and culture and sensitivity results, history of genitourinary abnormality, chronic medical conditions, previous history of UTI, place of visit (primary care clinic, or ED), and history of antibiotic prescriptions in last 6 months.

All urinary tract isolates of $E$. coli were identified and subjected to susceptibility testing with the Vitek 2 automated system (Bio Merieux Vitek, Inc., Hazelwood, MO). Vitek 2 is an automated computerized instrument which provides specific quantitative results of urine cultures more rapidly when compared to conventional methods. It is highly sensitive (92.8\%), and specific (99.4\%) with average predictive value of $92 \%$ [11]. The breakpoints (microgram/mL) for $E$. coli isolates were based on Clinical Laboratory Standard Institute guidelines formerly known as the National Committee on Clinical Laboratory Standards [12]. The data set was then limited to the first isolate tested for antibiotic susceptibilities per patient to minimize potential bias resulting from repeat cultures.

\section{Statistical Analysis}

SAS Enterprise Guide for Windows Version 4.3 statistical software was used for data analysis. Summary statistics were performed for frequencies and proportions for categorical variables. Univariate statistics were calculated using $\chi^{2}$ test at 5\% significance level. Univariate and multivariate logistic regression models were analyzed to determine the association of predictor variables with commonly prescribed antibiotics. Results are presented as odds ratios (ORs) with $95 \%$ confidence intervals (Cls).

\section{Results}

During the study period 5,621 urinary tract isolates of $E$. coli from 4,236 different patients were examined for susceptibility patterns. The median patient age was 41-years-old and body mass index (BMI) was 27.8. Overall, $37.7 \%$ E. coli isolates were resistant to ampicillin, $18.3 \%$ to TMP/SMX, $13.5 \%$ to ampicillin/sulbactam, $7.8 \%$ to Cipro/levofloxacin, and $3.8 \%$ to cefazolin (Table 1). Ciprofloxacin and Levofloxacin reported identical susceptibility pattern in the study urinary isolates. Therefore, only ciprofloxacin was used in further analy-

Table 1: Antimicrobial susceptibility results for Escherichia coli urinary isolates.

\begin{tabular}{|l|l|l|l|}
\hline Antimicrobial Agents & Total Number of isolates & Number of Isolates (\%) & \\
\hline & & Resistant & Susceptible/Intermediate \\
\hline Ampicillin & 5609 & $2115(37.7)$ & $3494(62.3)$ \\
\hline Ciprofloxacin & 5602 & $438(7.8)$ & $5164(92.2)$ \\
\hline Cefazolin & 5611 & $214(3.8)$ & $5397(96.2)$ \\
\hline Ampicillin/sulbactam & 5599 & $758(13.5)$ & $4841(86.5)$ \\
\hline Nitrofurantoin & 5611 & $82(1.5)$ & $5529(98.5)$ \\
\hline Levofloxacin & 5602 & $436(7.8)$ & $5166(92.2)$ \\
\hline TMP/SMX & 5611 & $1027(18.3)$ & $4584(81.7)$ \\
\hline
\end{tabular}

Abbreviation: TMP/SMX: Trimethoprim/Sulfamethoxazole. 
Table 2: Resistance to 1 or more antibiotics among 5,600 Escherichia coli urinary isolates against commonly prescribed antimicrobials.

\begin{tabular}{|c|c|c|c|c|c|c|}
\hline \multirow{2}{*}{$\begin{array}{l}\text { Number of agents to which } \\
\text { isolates were resistant }\end{array}$} & \multirow{2}{*}{$\begin{array}{l}\text { Total no. of } \\
\text { isolates (\%) }\end{array}$} & \multicolumn{5}{|c|}{ No. of Isolates (\%) resistant to } \\
\hline & & Ampicillin & TMP/SMX & Cefazolin & Nitrofurantoin & Ciprofloxacin \\
\hline 0 & $3,276(58.5)$ & & & & & \\
\hline 1 & $1,164(20.8)$ & $995(85.5)$ & $102(8.8)$ & $1(0.1)$ & $23(2.0)$ & $43(3.7)$ \\
\hline 2 & $836(14.9)$ & $794(95.0)$ & $644(77.0)$ & $83(9.9)$ & $20(2.4)$ & $131(15.7)$ \\
\hline $3^{*}$ & $266(4.8)$ & $266(100.0)$ & $224(84.2)$ & $75(28.2)$ & $26(9.8)$ & $207(77.8)$ \\
\hline $4^{*}$ & $52(0.9)$ & $52(100.0)$ & $51(98.1)$ & $48(92.3)$ & $6(11.5)$ & $51(98.1)$ \\
\hline $5^{*}$ & $6(0.1)$ & $6(100.0)$ & $6(100.0)$ & $6(100.0)$ & $6(100.0)$ & $6(100.0)$ \\
\hline
\end{tabular}

Abbreviation: TMP/SMX: Trimethoprim/Sulfamethoxazole; *: In all, 5.8\% (324 of 5600) of isolates were resistant to three or more antibiotics among the listed 5 antibiotics and defined as multidrug resistant.

Table 3: Factors associated with resistance to TMP/SMX and Ciprofloxacin Escherichia coli urinary isolates.

\begin{tabular}{|c|c|c|c|c|c|c|c|c|}
\hline \multirow{3}{*}{ Risk factors } & \multicolumn{4}{|l|}{ TMP/SMX } & \multicolumn{4}{|c|}{ Ciprofloxacin } \\
\hline & \multicolumn{2}{|c|}{ No. (\%) of isolates } & \multirow{2}{*}{ OR (95\% Cl) } & \multirow{2}{*}{$P$ value } & \multicolumn{2}{|c|}{ No. $(\%)$ of isolates } & \multirow{2}{*}{ OR $(95 \% \mathrm{CI})$} & \multirow{2}{*}{$P$ value } \\
\hline & $\mathbf{S}$ & $\mathbf{R}$ & & & $\mathbf{S}$ & $\mathbf{R}$ & & \\
\hline Overall & $4584(81.7)$ & $1027(18.3)$ & & & $5164(92.2)$ & $438(7.8)$ & & \\
\hline \multicolumn{9}{|l|}{ Gender } \\
\hline Male & $274(6.0)$ & $79(7.7)$ & $1.32(1.01-1.69)$ & 0.04 & $308(6.0)$ & $44(10.0)$ & $1.75(1.27-2.44)$ & 0.0008 \\
\hline Female & $4310(94.0)$ & $948(92.3)$ & 1 & & $4856(94.0)$ & $394(90.0)$ & 1 & \\
\hline \multicolumn{9}{|l|}{ Race } \\
\hline Asian & $70(1.5)$ & $43(4.2)$ & $3.15(2.14-4.65)$ & $<0.0001$ & $101(2.0)$ & $12(2.7)$ & $1.53(0.83-2.82)$ & 0.17 \\
\hline Black & $480(10.5)$ & $142(13.8)$ & $1.52(1.24-1.86)$ & $<0.0001$ & $550(10.6)$ & $71(16.2)$ & $1.67(1.27-2.19)$ & 0.0002 \\
\hline White & $3773(82.3)$ & $735(71.6)$ & 1 & & 4178 (80.9) & $324(74.0)$ & 1 & \\
\hline \multicolumn{9}{|l|}{ Health Insurance } \\
\hline Yes & $4255(92.8)$ & $957(93.2)$ & $1.06(0.81-1.38)$ & 0.68 & 4795 (92.9) & $408(93.2)$ & $1.05(0.71-1.54)$ & 0.82 \\
\hline No & $329(7.2)$ & $70(6.8)$ & 1 & & $369(7.1)$ & $30(6.8)$ & 1 & \\
\hline \multicolumn{9}{|l|}{ Location } \\
\hline $\begin{array}{l}\text { Emergency } \\
\text { Department }\end{array}$ & $530(11.6)$ & $152(14.8)$ & $1.08(0.79-1.46)$ & 0.64 & $614(11.9)$ & $68(15.5)$ & $0.36(0.26-0.51)$ & $<0.0001$ \\
\hline Outpatient & 3754 (81.9) & $795(77.4)$ & $0.79(0.61-1.03)$ & 0.08 & $4259(82.5)$ & $281(64.2)$ & $0.22(0.17-0.28)$ & $<0.0001$ \\
\hline Inpatient & $300(6.5)$ & $80(7.8)$ & 1 & & $291(5.6)$ & $89(20.3)$ & 1 & \\
\hline \multicolumn{9}{|c|}{ History of Genitourinary Abnormality } \\
\hline Yes & $347(7.6)$ & $118(11.5)$ & $1.59(1.27-1.98)$ & $<0.0001$ & $393(7.6)$ & $71(16.2)$ & $2.35(1.79-3.09)$ & $<0.0001$ \\
\hline No & $4237(92.4)$ & $909(88.5)$ & 1 & & $4771(92.4)$ & $367(83.8)$ & 1 & \\
\hline \multicolumn{9}{|c|}{ History of Chronic Medication } \\
\hline Yes & $217(4.7)$ & $56(5.5)$ & $1.16(0.86-1.57)$ & 0.33 & $235(4.6)$ & $38(8.7)$ & $1.99(1.39-2.85)$ & 0.0002 \\
\hline No & 4367 (95.3) & $971(94.5)$ & 1 & & $4929(95.4)$ & $400(91.3)$ & 1 & \\
\hline \multicolumn{9}{|c|}{ Mode of Urine Collection } \\
\hline Catheterized & $170(3.7)$ & $73(7.1)$ & $1.99(1.50-2.64)$ & $<0.0001$ & $175(3.4)$ & $68(15.5)$ & $5.24(3.89-7.07)$ & $<0.0001$ \\
\hline Void & $4414(96.3)$ & $954(92.9)$ & 1 & & 4989 (96.6) & $370(84.5)$ & 1 & \\
\hline \multicolumn{9}{|l|}{ Diabetes } \\
\hline Yes & $579(12.6)$ & $170(16.6)$ & $1.37(1.14-1.65)$ & 0.0009 & $635(12.3)$ & $114(26.0)$ & $2.51(2.00-3.16)$ & $<0.0001$ \\
\hline No & $4005(87.4)$ & $858(83.4)$ & 1 & & $4529(87.7)$ & $324(74.0)$ & 1 & \\
\hline \multicolumn{9}{|l|}{ Cancer } \\
\hline Yes & $308(6.7)$ & $69(6.7)$ & $1.00(0.76-1.31)$ & 1 & $316(6.1)$ & $61(13.9)$ & $2.48(1.85-3.33)$ & $<0.0001$ \\
\hline No & $4276(93.3)$ & $958(93.3)$ & 1 & & 4848 (93.9) & $377(86.1)$ & 1 & \\
\hline Age & & & $0.99(0.99-1.00)$ & 0.0036 & & & $1.03(1.02-1.04)$ & $<0.0001$ \\
\hline \multicolumn{9}{|c|}{ Previous Diagnosis of UTI } \\
\hline 0 (reference) & $2630(57.4)$ & $532(51.8)$ & 1 & & $2996(58.0)$ & $160(36.5)$ & 1 & \\
\hline 1 & $944(20.6)$ & $182(17.7)$ & $0.95(0.79-1.15)$ & 0.61 & $1044(20.2)$ & $80(18.3)$ & $1.44(1.09-1.89)$ & 0.01 \\
\hline 2 & $298(8.7)$ & $92(9.0)$ & $1.14(0.89-1.46)$ & 0.29 & $446(8.6)$ & $44(10.1)$ & $1.85(1.30-2.62)$ & 0.0006 \\
\hline 3 & $194(4.2)$ & $56(5.5)$ & $1.43(1.05-1.95)$ & 0.025 & $224(4.3)$ & $26(5.9)$ & $2.17(1.41-3.36)$ & 0.0005 \\
\hline 4 & $111(2.4)$ & $40(3.9)$ & $1.78(1.23-2.59)$ & 0.0024 & $130(2.5)$ & $20(4.6)$ & $2.88(1.75-4.74)$ & $<0.0001$ \\
\hline 05-Oct & $213(4.7)$ & $82(8.0)$ & $1.90(1.45-2.50)$ & $<0.0001$ & $239(4.6)$ & $56(12.8)$ & $4.39(3.15-6.11)$ & $<0.0001$ \\
\hline$>10$ & $94(2.1)$ & $43(4.2)$ & $2.26(1.56-3.28)$ & $<0.0001$ & $85(1.7)$ & $52(11.9)$ & $11.46(7.83-16.75)$ & $<0.0001$ \\
\hline \multicolumn{9}{|l|}{ Hospitalization } \\
\hline 0 (reference) & 4415 (96.3) & $966(94.1)$ & 1 & & $5016(97.1)$ & $357(81.5)$ & 1 & \\
\hline 1 & $102(2.2)$ & $38(3.7)$ & $1.70(1.17-2.49)$ & 0.006 & $89(1.7)$ & $51(11.6)$ & $8.05(5.62-11.55)$ & $<0.0001$ \\
\hline$\geq 2$ & $67(1.5)$ & $23(2.2)$ & $1.57(0.97-2.53)$ & 0.07 & $59(1.1)$ & $30(6.9)$ & $7.14(4.54-11.23)$ & $<0.0001$ \\
\hline
\end{tabular}




\begin{tabular}{|c|c|c|c|c|c|c|c|c|}
\hline \multicolumn{9}{|l|}{ Obesity } \\
\hline $\mathrm{BMI}<25$ & $1887(41.2)$ & $422(41.1)$ & 1 & & $2157(41.8)$ & $148(33.8)$ & 1 & \\
\hline BMI 25-29.9 & $1074(23.4)$ & $227(22.1)$ & $0.95(0.79-1.13)$ & 0.53 & $1215(23.5)$ & $84(19.2)$ & $1.01(0.76-1.33)$ & 0.96 \\
\hline $\mathrm{BMI} \geq 30$ & $1623(35.4)$ & $378(36.8)$ & $1.04(0.89-1.22)$ & 0.61 & $1792(34.7)$ & $206(47.0)$ & 1.68 (1.34-2.09) & $<0.0001$ \\
\hline \multicolumn{9}{|c|}{ Antibiotic Prescribed in Past 6 Months } \\
\hline 0 (reference) & $1190(26.0)$ & $185(18.0)$ & 1 & & $1302(25.2)$ & $71(16.2)$ & 1 & \\
\hline 1 & $1698(37.0)$ & $321(31.3)$ & $1.22(1.00-1.48)$ & 0.05 & $1930(37.4)$ & $85(19.4)$ & $0.81(0.59-1.12)$ & 0.19 \\
\hline 02-Mar & $1151(25.1)$ & $307(29.9)$ & $1.72(1.41-2.10)$ & $<0.0001$ & $1334(25.8)$ & $122(27.9)$ & $1.68(1.24-2.27)$ & 0.0008 \\
\hline 04-May & $310(6.8)$ & $107(10.4)$ & $2.22(1.70-2.91)$ & $<0.0001$ & $361(7.0)$ & $56(12.8)$ & $2.85(1.97-4.12)$ & $<0.0001$ \\
\hline$>5$ & $235(5.1)$ & $107(10.4)$ & $2.93(2.22-3.86)$ & $<0.0001$ & $237(4.6)$ & $104(23.7)$ & $8.05(5.77-11.22)$ & $<0.0001$ \\
\hline
\end{tabular}

Abbreviation: TMP/SMX: Trimethoprim/Sulfamethoxazole; OR: Odds ratio.

ses. When multidrug resistance (MDR) rates were analyzed among the 5 most commonly prescribed antibiotics, $58.5 \%$ of total isolates were susceptible to all antimicrobials and $20.8 \%$ were resistant to at least 1 antimicrobial agent (Table 2). Approximately $15 \%$ urinary isolates were resistant to at least 2 agents, predominantly ampicillin (95\%), and TMP/SMX (77\%) followed by Ciprofloxacin (15.7\%). MDR was defined if urinary isolates were resistant to at least one agent in three or more antimicrobial categories [13]. MDR isolates accounted for 5.8\% ( $n$ $=324$ ) of 5,600 isolates. The majority of MDR isolates were resistant to 3 antimicrobials, and these accounted for $4.8 \%(n=266)$ of all isolates. Among all the MDR phenotypes, $82.1 \%(n=266)$ isolates were concurrently resistant to at least $3 \geq$ antibiotics.

When the risk of resistance to TMP/SMX and ciprofloxacin in relation to patient demographics and clinical variables were analyzed, men who were infected with $E$. coli uropathogen were found to be at increased risk for developing resistance to these antibiotics compared to women (Table 3). Black patients were at a significant risk for developing resistance to TMP/SMX $(O R=1.52$, $C l$ 1.24-1.86), and ciprofloxacin (OR = 1.67, $\mathrm{Cl}$ 1.27-2.19) than their white counterparts. The odds of risk of resistance in Asians than to whites was significant for TMP/ $\operatorname{SMX}(O R=3.15, \mathrm{Cl} 2.14-4.65)$ but not for ciprofloxacin. Compared to inpatient, ciprofloxacin resistance was significantly lower for $E$. coli urinary isolates collected either from outpatient clinics $(O R=0.36, \mathrm{Cl} 0.26-0.51)$ or $\operatorname{ED}(O R=0.22, \mathrm{Cl} 0.17-0.28)$. Urine samples collected via catheterization were significantly associated with $E$. coli resistance antibiotics; SMP/TMX: $O R=1.99,(C l 1.50$ 2.64); and ciprofloxacin: $O R=5.24,(C l$ 3.89-7.07) than voided specimens. Patients with a history of genitourinary abnormality were 1.6 and 2.3 times more likely to have urinary isolates resistant to TMP/SMX $(P<0.0001)$ and ciprofloxacin $(P<0.0001)$, respectively. Patients who were on chronic medications $(P<0.0002)$ or who had cancer $(P<0.0001)$ were likely to have ciprofloxacin resistant $E$. coli urinary isolates than otherwise healthy adults. Compared to non-diabetic patients $E$. coli isolates from patients with diabetes appeared to be 2.51 times ( $\mathrm{Cl}$ 2.00-3.16) more likely to be resistant to ciprofloxacin, and 1.37 times ( $\mathrm{Cl} 1.14-1.65)$ to TMP/SMX. Although not significant, an overall increasing trend in resistance was observed against both antimicrobials when isolates were compared for overweight (BMI 25-29.9) and obese $(\mathrm{BMI} \geq 30$ ) patients to patients whom $\mathrm{BMI}$ was $<25$. For ciprofloxacin, the odds of resistance were significantly higher among obese patients $(O R=1.68, \mathrm{Cl} 1.34-2.09)$. When ordinal variables were considered including previous diagnosis of UTIs, numbers of hospitalization and antibiotics prescribed in past 6 months, a clear increasing trend in risk of developing resistance against $E$. coli uropathogen was observed for both antibiotics. For patients who had 5-10 episodes of UTIs or who had $\geq 11$ episodes of UTI, the odds of resistance increased to 1.90 $(P<0.0001)$ and $2.26(P<0.0001)$, respectively for TMP/ SMX, and $4.39(P<0.0001)$ and $11.46(P<0.0001)$ for ciprofloxacin. Similarly, the risk of resistance to these two antimicrobials gradually increased among patients who were hospitalized $\geq 2$ times (ciprofloxacin: $O R=7.14, \mathrm{Cl}$ 4.54-11.23 TMP/SMX: OR = 1.57, $\mathrm{Cl}$ 0.97-2.53) or who had $\geq 6$ antibiotics prescriptions in last 6 months (ciprofloxacin: $O R=8.05, C l$ 5.77-11.22 TMP/SMX: $O R=2.93$, Cl 2.22-3.86).

We have observed high collinearity among previous diagnosis of UTI, history of genitourinary abnormality, hospitalization, and antibiotic prescriptions in last 6 months. For instance, the Pearson correlation between hospitalization and previous diagnosis of UTI was 0.61 , and the correlation between hospitalization and antibiotic prescriptions in past six months was 0.41 . Such collinearity precludes us to use multivariate logistic regression including all variables simultaneously; however, when collinear variables were removed the results were similar to those from the univariate analysis.

\section{Discussion}

Both increasing trends in antimicrobial resistance of E. coli urinary isolates to $\mathrm{TMP} / \mathrm{SMX}$ and the potential subsequent decreases in its efficacy as empiric therapy in treating uncomplicated UTIs in ambulatory care settings pose a serious challenge to our health care providers. Such resistance patterns and revised recommendations from the IDSA compel health care providers to take into consideration alternative therapies, like fluoroquinolones or nitrofurantoin. Therefore, knowledge of the multi-antimicrobial resistance pattern of commonly prescribed antibiotics and the associated risk factors in developing resistance in the local community is critical for practitioners to empirically select an effective 
therapeutic agent and thereby reduce the risk of treatment failure.

Overall in vitro antimicrobial resistance rates among $E$. coli urinary isolates in our study is similar to the national benchmark reported in previous studies [14,15]. For example, except for ciprofloxacin, the resistances to ampicillin (37.7\% vs. 39.1\%) and TMP/SMX (18.3\% vs. $18.6 \%)$ are consistent with the resistance prevalence reported in the USA 2000 national prevalence data used by Sahm, et al. [15]. Compared to that study the resistance rate has nearly doubled (3.7\% to $7.8 \%$ ) for ciprofloxacin while the TMP/SMX resistance rate has remained the same at $18 \%$. We have also observed a marginal increase in nitrofurantoin resistance from $1 \%$ to $1.5 \%$. Ciprofloxacin resistance in our study rose sharply from $15.7 \%$ to $77.8 \%$ compared to ampicillin (95\% to $100 \%)$ and TMP/SMX (77\% to $84.2 \%)$ when 3 antimicrobials phenotypes were considered (Table 2). Increase in provider use of fluoroquinolones may have contributed to rapid rising in antimicrobial resistance of $E$. coli to ciprofloxacin, as resistance to this agent has shown to correlate with the level of its use $[16,17]$.

While previous retrospective studies that have inferred co-resistance of ciprofloxacin with other antimicrobials are unlikely in outpatient urinary E. coli isolates, our susceptibility data reported about 38\% ( $n=$ $437 / 1160$ ) of all MDR phenotypes which are resistant to ciprofloxacin were also resistant to 4 common antimicrobial phenotypes $[6,15,18,19]$. The co-resistance association between the ciprofloxacin resistant isolates with isolates resistant for TMP/SMX, nitrofurantoin, and cefazolins appeared to be significantly $(P<0.0001)$ strong (Table 4 and Table 5). Nearly $58 \%$ percent of cipro-resistant $E$. coli isolates were also resistant to TMP/ SMX, $81 \%$ to ampicillin, $20 \%$ to cefazolin, and 5\% to nitrofurantoin. The findings are in agreement with previous reports suggesting fluoroquinolones resistance typically arises in isolates of $E$. coli which already har-

Table 4: Association between ciprofloxacin and four common antibiotics.

\begin{tabular}{|c|c|c|c|c|}
\hline & \multirow[t]{2}{*}{$\begin{array}{l}\text { No. of } \\
\text { Isolates }\end{array}$} & \multicolumn{2}{|c|}{ Ciprofloxacin No. (\%) } & \multirow[t]{2}{*}{$p$-value } \\
\hline & & $\mathbf{S}^{*}$ & $\mathbf{R}^{* *}$ & \\
\hline \multicolumn{5}{|c|}{ TMP/SMX } \\
\hline S & 4,575 & $4,392(85.1)$ & $183(41.8)$ & $<0.0001$ \\
\hline $\mathrm{R}$ & 1,027 & $772(14.9)$ & $255(58.2)$ & \\
\hline \multicolumn{5}{|c|}{ Ampicillin } \\
\hline$S$ & 3,487 & $3,406(66.0)$ & $81(18.5)$ & $<0.00001$ \\
\hline $\mathrm{R}$ & 2,113 & $1,756(34.0)$ & $357(81.5)$ & \\
\hline \multicolumn{5}{|c|}{ Nitrofurantoin } \\
\hline$S$ & 5,520 & $5,106(98.9)$ & $414(94.5)$ & $<0.0001$ \\
\hline $\mathrm{R}$ & 82 & $58(1.1)$ & $24(5.5)$ & \\
\hline \multicolumn{5}{|c|}{ Cefazolin } \\
\hline$S$ & 5,388 & $5,036(97.5)$ & $352(80.4)$ & $<0.0001$ \\
\hline $\mathrm{R}$ & 214 & $128(2.5)$ & $86(19.6)$ & \\
\hline
\end{tabular}

Abbreviation: TMP/SMX: Trimethoprim/Sulfamethoxazole; $\mathrm{S}^{*}$ : Susceptible; $\mathrm{R}^{* *}$ : Resistant. bor ampicillin and/or TMP/SMX resistant mutants. The clonal expansion of MDR isolates may be amplified by exposure to any single agent for which resistance exists. Two recent European studies reported a strong positive correlation between the ciprofloxacin usage and the proportion of TMP/SMX resistance $E$. coli per year suggesting that the resistance to TMP/SMX might have been induced by ciprofloxacin treatment [20,21].

The activity of fluoroquinolones and nitrofurantoin against $E$. coli resistant uncomplicated UTIs may be of important consideration in communities where the TMP/ SMX resistance rate exceeds the IDSA recommended threshold (20\%). We have found concurrent resistance of ciprofloxacin about 8 times more frequent $(24.8 \%$ vs. $3.1 \%)$ than resistance to nitrofurantoin among TMP/ SMX-resistance $E$. coli urinary isolates (Table 5) which is in line with the findings from Karlowsky, et al. (9.5\% vs. $1.7 \%$, about 5 times more common) [18]. Yet, this apparent difference is minimal when overall susceptibility rate for ciprofloxacin (92.2\%) cefazolin (96.2\%), and nitrofurantoin (98.5\%) were considered in our study. Furthermore, among the TMP/SMX-resistant $E$. coli urinary isolates $75 \%$ of the specimens were still sensitive to ciprofloxacin, $91 \%$ to cefazolin, and $97 \%$ to nitrofurantoin. Therefore, longitudinal monitoring of the course of TMP/ SMX resistance as well as the susceptibility pattern of ciprofloxacin and nitrofurantoin are important as these alternative therapies become more widely prescribed.

Caution must be considered, however, when choosing ciprofloxacin over nitrofurantoin or vice versa. There was $5.5 \%$ of ciprofloxacin-resistant urinary isolates which were also resistant to nitrofurantoin. On the other hand, $29.3 \%$ of the nitrofurantoin-resistant isolates were resistant to ciprofloxacin. It was $10.4 \%$ vs. $29.8 \%$ in the Karlowsky, et al. study [18]. Hence, emphasizing the rationality of prescribing nitrofurantoin over ciprofloxacin as a second-line agent when TMP/SMX cannot be used for uncomplicated UTIs. Unlike ciprofloxacin

Table 5: Association between TMP/SMX and four common antibiotics.

\begin{tabular}{|c|c|c|c|c|}
\hline \multirow{2}{*}{ No. of Isolates } & & \multicolumn{2}{|c|}{ TMP/SMX No. (\%) } & \multirow{2}{*}{$p$-value } \\
\hline & & $\mathbf{S}^{*}$ & $\mathbf{R}^{* *}$ & \\
\hline \multicolumn{5}{|l|}{ Ciprofloxacin } \\
\hline$S$ & 5164 & $4,392(96.0)$ & $772(75.2)$ & $<0.0001$ \\
\hline $\mathrm{R}$ & 438 & $183(4.0)$ & $255(24.8)$ & \\
\hline \multicolumn{5}{|l|}{ Ampicillin } \\
\hline$S$ & 3,494 & $3,354(73.2)$ & $140(13.6)$ & $<0.0001$ \\
\hline $\mathrm{R}$ & 2,115 & $1,228(26.8)$ & $887(86.4)$ & \\
\hline \multicolumn{5}{|l|}{ Nitrofurantoin } \\
\hline$S$ & 5,529 & $4,534(98.9)$ & 995 (96.9) & $<0.0001$ \\
\hline $\mathrm{R}$ & 82 & $50(1.1)$ & $32(3.1)$ & \\
\hline \multicolumn{5}{|l|}{ Cefazolin } \\
\hline$S$ & 5,397 & $4,465(97.4)$ & $932(90.7)$ & $<0.0001$ \\
\hline $\mathrm{R}$ & 214 & $119(2.6)$ & $95(9.3)$ & \\
\hline
\end{tabular}

Abbreviation: TMP/SMX: Trimethoprim/Sulfamethoxazole; S*: Susceptible; $\mathrm{R}^{* *}$ : Resistant. 
and cefazolin which are broader in spectrum and have a wide variety of indications, nitrofurantoin is a unique drug for treatment of uncomplicated UTIs in outpatient areas because of its narrow spectrum bactericidal activity and its limited contact with bacteria outside the urinary tract. B-lactams, including second generation cephalosporin, are generally less effective and have more adverse effects. Therefore, they are not an option for first-line treatment for uncomplicated UTIs.

Our study also reported men are at significant risk of developing resistant $E$. coli urinary isolates to TMP/ SMX and ciprofloxacin than women. A similar trend was observed in our pediatric study as well as the NAUTICA surveillance study of outpatient UTIs [1,14]. The NAUTICA study reported greater antibiotic resistance to ciprofloxacin, and TMP/SMX among all urinary isolates from US and Canadian male patients. Despite the fact of higher prevalence of UTIs among women due to their anatomic and physiologic factors, the etiology of men being more prone to have antibiotic resistance $E$. coli isolates was not clearly understood. One likely explanation could be men are likely to present with complicated UTIs hence more at risk of developing antimicrobial resistant pathogens. In addition to patient age, gender, and race, we found a strong association and trend in developing TMP/SMX and ciprofloxacin resistant isolates with increasing frequency of UTI infections and number of antibiotic prescriptions filled in last 6 months. Ciprofloxacin resistant risk increased exponentially for patients who had $>4$ UTIs or had $>5$ antibiotic prescriptions in last 6 months. Patients' age, severity of illness, history of multiple hospitalizations, uropathological disorders, comorbidities including obesity, and diabetes are potential explanations for developing such resistance $[1,3,6,7]$.

Studies have been equivocal in finding the role of diabetes as independent risk factors for $E$. coli resistance isolates, particularly in ambulatory care settings [22-24]. In our study diabetes remained strong risk factors for $\mathrm{TMP} / \mathrm{SMX}(O R=1.39 ; P<0.001)$, and ciprofloxacin $(O R$ $=2.03 ; P<0.0001)$ even when urinary isolates were excluded for patients who were admitted in hospital from ambulatory care clinics and ED. Advanced age, poor compliance, frequent infections leading to more use of antibiotics, and hospitalization could be some of the mechanisms by which this subgroup of population acquires resistant uropathogens.

Unlike other studies, our study also found a significant association between increasing antimicrobial trend in resistance to TMP/SMX and ciprofloxacin with obesity. The data clearly indicates the ordinal increase in risk of developing resistance when BMI goes over 25. In particular, the risk was found to be significantly high for ampicillin and ciprofloxacin. A complex relationship has been suggested in literature among dietary habit, use of frequent antimicrobials leading to change in gut flora which ultimately contributes to increasing the risk of developing obesity later in life [25-28]. Although the actual causality and the temporal associations among these variables remain to be elucidated, repeated or early exposure of antibiotics might be playing a critical role in modulation of intestinal microbes which in turn influence host metabolism and lead to fat accumulation.

Contrasting surveillance data provides a snap shot of antimicrobial susceptibility patterns of different geographical locations; the current study strength is that it is uniquely able to examine the relationship of demographical and clinical variables as risk factors against antimicrobials at an individual level. Patient age, gender, race, previous history of UTIs, number of antibiotic prescriptions in the last 6 months, and comorbidities, such as obesity and diabetes, are found to be independent risk factors for antimicrobial resistance to urinary $E$. coli infections. The study was also able to evaluate the resistant and concurrent resistant patterns of different antimicrobials at outpatient community settings. All urinary samples were analyzed in one central location and the laboratory data was merged with an electronic clinical dataset which was later validated systematically with individual patients' medical records.

The results of our investigation, however, must be interpreted in light of the following considerations. When a narrower spectrum agent would suffice, empiric broad spectrum antibiotic selection, without routine urine cultures, are common health care practice for patients with uncomplicated UTI. Hence, there is a potential for overestimating the resistance rates in the community by selectively ordering urine cultures for patients whose UTIs are either serious, recurrent, not responding to empiric therapy, or requiring hospitalization. Despite our efforts in collaborating urinary isolates data systematically with patient demographics and clinical history, certain family history such as history of hospitalization or antimicrobial use in a family member, contact with pets and livestock, having a child in daycare, and dietary habits information are deficient. While the study results are useful to our health care practitioners at the local level, caution must be made for its applicability to other geographic areas.

In summary, our study demonstrates the importance of monitoring TMP/SMX, ciprofloxacin, and nitrofurantoin susceptibility patterns and the association of MDR phenotypes in E. coli urinary isolates. Because the emergence of resistance to ciprofloxacin and its propensity of inducing co-resistance to nitrofurantoin, and TMP/ SMX phenotypes, the use of ciprofloxacin should be discouraged as it will undermine the efficacy in treating more serious infections. Alternative antimicrobials, such as nitrofurantoin which is narrow in coverage and mostly stays in urinary tract, should be preferred in the treatment of TMP/SMX-resistant UTIs (exceeds 20\%). Further longitudinal studies are warranted to identify 
specific risk factors and virulence of organisms at broader geographic locations for better understanding of the potential forces that trigger the resistance. Meanwhile, a multidisciplinary, educational partnership incorporating patients, health care providers, and local leaders are recommended at community and national levels to promote judicious use of antimicrobials in order to prolong the clinical effectiveness of existing agents.

\section{Acknowledgement}

This study was approved by Carle Institutional Review Board.

We thank Dr. Peiyong Qu from the University of IIlinois Statistical Department for helping with statistical analysis and Dr. Jennifer Eardley from the Stephens Family Clinical Research Institute for logistical support in conducting this study.

\section{Declaration of Conflicting Interests}

The authors declare no conflicts of interest with respect to the authorship and/or publication of this article.

The study was approved by local Carle Institutional Review Board.

\section{Funding}

The authors disclose receipt of following financial support for the research and/or authorship of this article.

This study was supported by the Stephens Family Clinical Research Institute.

\section{References}

1. Ahmed MN, Vannoy D, Frederick A, Chang S, Lawler E (2016) First-line antimicrobial resistance patterns of Escherichia Coli in children with urinary tract infection in emergency department and primary care clinics. Clin Pediatr (Phila) 55: 19-28.

2. Gupta K, Scholes D, Satmm WE (1999) Increasing prevalence of antimicrobial resistance among uropathogens causing acute uncomplicated cystitis in young women. JAMA 281: 736-738.

3. Gales AC, Jones RN, Gordon KA, Sader HS, Wilke WW, et al. (2000) Activity and spectrum of 22 antimicrobial agents tested against urinary tract infection pathogens in hospitalized patients in Latin America: report from the second year of the SENTRY antimicrobial surveillance program (1998). J Antimicrob Chemother 45: 295-303.

4. Jones RN, Kugler KC, Pfaller MA, WinoKur PI (1999) Characteristics of pathogens causing urinary tract infections in hospitals in North America: results from the SENTRY antimicrobial surveillance program, 1997. Diagn Microbiol Infect Dis 35: 55-63.

5. Sanchez GV, Master RN, Karlowsky JA, Bordon JM (2012) In vitro antimicrobial resistance of urinary Escherichia Coli isolates among U.S. outpatients from 2000 to 2010. J Antimicrob Chemother 56: 2181-2183.

6. Zhanel GG, Karlowsky JA, Harding GK, Carrie A, MazzuIli T, et al. (2000) A Canadian national surveillance study of urinary tract isolates from outpatients: comparison of the activities of trimethoprim-sulfamethoxazole, ampicillin, mecillinam, nitrofurantoin, and ciprofloxacin. Antimicrob Agents Chemother 44: 1089-1092.
7. Gupta K, Hooton TM, Naber KG, Wult B, Colgan R, et al. (2011) International clinical practice guidelines for the treatment of acute uncomplicated cystitis and pyelonephritis in women: a 2010 update by the Infectious Diseases Society of America and the European Society for Microbiology and Infectious Diseases. Clin Infect Dis 52: e103-e120.

8. McCaig LF, Besser RE, Hughes JM (2003) Antimicrobial-drug prescription in ambulatory care settings, United States, 1992-2000. Emerg Infec Dis 9: 432-437.

9. Huang ES, Stafford RS (2002) National patterns in the treatment of urinary tract infections in women by ambulatory care physicians. Arch Intern Med 162: 41-47.

10. Gupta K, Hooton TM, Stamm WE (2001) Increasing antimicrobial resistance and the management of uncomplicated community-acquired urinary tract infections. Ann Intern Med 135: 41-50.

11. Smith PB, Gavan TL, Isenberg HD, Sonnenwirth A, Taylor WI, et al. (1978) Multi-laboratory evaluation of an automated microbial detection/identification system. J Clin Microbiol 8: 657-666.

12. Clinical and Laboratory Standards Institute (2009) Methods for dilution antimicrobial susceptibility tests for bacteria that grow aerobically. Approved standard, 8th ed. CLSI publication M07-A8. Clinical and Laboratory Standards Institute, Wayne, PA.

13. Magiorakos AP, Srinivasan A, Carey RB, Carmeli Y, Falagas ME, et al. (2012) Multidrug-resistant, extensively drug-resistance and pandrug-resistant bacteria: an international expert proposal for interim standard definitions for acquired resistance. Clin Microbiol Infect 18: 268-281.

14. Zhanel GG, Hisanaga TL, Laing NM, DeCorby MR, Nichol $\mathrm{KA}$, et al. (2006) Antibiotic resistance in Escherichia coli outpatient urinary isolates: final results from the North American Urinary Tract Infection Collaborative Alliance (NAUTICA). Int J Antimicrob Agents 27: 468-475.

15. Sahm DF, Thornsberry C, Mayfield DC, Jones ME, Karlowsky JA (2001) Multidrug-resistance urinary tract isolates of Escherichia Coli: prevalence and patient demographics in the United States in 2000. Antimicrob Agents Chemother 45: 1402-1406.

16. Johnson L, Sabel A, Burman WJ, Everhart RM, Rome M, et al. (2008) Emergence of fluoroquinolone resistance in outpatient Escherichia Coli urinary isolates. Am J Med 121: 876-884.

17. Zervos MJ, Hershberger E, Nicolau DP, Ritchie DJ, Blackner LK, et al. (2003) Relationship between fluoroquinolone use and changes in susceptibility to fluoroquinolones of selected pathogens in 10 United States teaching hospitals. Clin Infec Dis 37: 1643-1648.

18. Karlowsky JA, Thornsberry AC, Jones ME, Sahm DF (2003) Susceptibility of antimicrobial-resistant urinary isolates Escherichia Coli to fluoroquinolones and nitrofurantoin. Clin Infect Dis 36: 183-187.

19. Lautenbach E, Strom BL, Bilker WB, Patel JB, Edelstein $\mathrm{PH}$, et al. (2001) Epidemiological investigation of fluoroquinolone resistance in infections due to extended spectrum $\beta$-lactamase producing Escherichia Coli and Klebsiella pneumoniae. Clin Infect Dis 33: 1288-1294.

20. Farra A, Skoog G, Wallen L, Kahlmeter G, Kronvall G, et al. (2002) Antibiotic use and Escherichia Coli resistance trend for quinolones and cotrimoxazole in Sweden. Scand J Infect Dis 34: 449-455.

21. Borgmann S, Jakobiak T, Gruber H, Schroder H, Sagel U (2009) Ciprofloxacin treatment of urinary infections results 
in increased resistance of urinary $\mathrm{E}$. Coli to ciprofloxacin and co-trimoxazole. Pol J Microbiol 58: 371-373.

22. Wright SW, Wrenn KD, Haynes ML (1999) Trimethoprim-sulfamethoxazole resistance among urinary coliform isolates. J Gen Intern Med 14: 606-609.

23. Malmartel A, Ghasarossian C (2016) Bacterial resistance in urinary tract infections in patients with diabetes matched with patients without diabetes. J Diabetes Complications 30: 705-709.

24. Bonadio M, Costarelli S, Morelli G, Tartaglia T (2006) The influence of diabetes mellitus on the spectrum of uropathogens and the antimicrobial resistance in elderly adult patients with urinary tract infections. BMC Infec Dis 6: 54 .
25. Azad MB, Bridgman SL, Becker AB, Kozyrskyj AL (2014) Infant antibiotic exposure and the development of childhood overweight and central adiposity. Int J Obes 38: 1290-1298.

26. Bailey LC, Forrest CB, Zhang P, Richards TM, Livshits A, et al. (2014) Association of antibiotics in infancy with early childhood obesity. JAMA Pediatr 168: 1063-1069.

27. Turnbaugh PJ, Ley RE, Mahowald MA, Magrini V, Mardis ER, et al. (2006) An obesity-associated gut microbiome with increased capacity for energy harvest. Nature 444: 1027-1031.

28. Vael C, Verhulst SL, Nelen V, Goossens H, Desager KN (2011) Intestinal microflora and body mass index during the first three years of life: an observational study. Gut Pathog 3: 8. 